\title{
Indium oxide thin-film homo-junctions: Morphology and electrical properties
}

\author{
Jolanta Stankiewicz, ${ }^{1}$ Francisco Villuendas, ${ }^{2, a)}$ María Pilar Lozano, ${ }^{3}$ and Isabel Díez ${ }^{1}$ \\ ${ }^{1}$ Departamento de Física de la Materia Condensada, Instituto de Ciencia de Materiales de Aragón, \\ CSIC-Universidad de Zaragoza, 50009-Zaragoza, Spain \\ ${ }^{2}$ Departamento de Física Aplicada, Universidad de Zaragoza, 50009-Zaragoza, Spain \\ ${ }^{3}$ Instituto Tecnológico de la Construcción, 46980 Paterna-Valencia, Spain
}

(Received 6 May 2013; accepted 7 August 2013; published online 23 August 2013)

\begin{abstract}
Indium oxide shows an unusual combination of electrical and optical properties that give rise to a broad range of applications in optoelectronic devices. Here, we report results of structural, $\mathrm{x}$-ray photoelectron spectroscopy, and electrical transport studies of transparent homo-junctions, obtained by sequential growth of polycrystalline thin layers of indium oxide under $\mathrm{O}_{2}$-rich and $\mathrm{O}_{2}$-poor conditions. We find that the growth temperature, which affects significantly film morphology, is critical for the rectifying behavior of the junctions. Only junctions grown at about $350{ }^{\circ} \mathrm{C}$ are rectifying. We also find that $p$-type-like layers have higher concentration of inter-grain oxygen than $n$-type layers, presumably coming from oxygen-rich deposition conditions and from much larger number of grain boundaries than in $n$-type layers. We conjecture that the segregation of oxygen ions at grain boundaries is responsible for the formation of inversion layers in $\mathrm{O}_{2}$-rich films and their apparent $p$-type $d c$ conduction. This mechanism significantly modifies the capacitance-voltage characteristics of the junctions. However, the rectifying mechanism can be accounted for by a space-charge layer at the $p$ - $n$ interface. Such behavior might be important in other polycrystalline thin films with a large number of interface defects at grain boundaries. C 2013 AIP Publishing LLC. [http://dx.doi.org/10.1063/1.4819177]
\end{abstract}

\section{INTRODUCTION}

Transparent conducting oxide (TCO) films are broadly applied in present-day optoelectronic devices. They are used as transparent electrodes in flat panel displays, solar cells, touch panels, low emissivity windows, and gas sensors. ${ }^{1-5}$ Indium oxide, which is a direct wide gap semiconductor, is one of the most widely applied TCO materials. Studies of its properties have mainly focused on heavily doped $n$-type films in search of highly transparent material with nearly metallic conductivity. This goal seems to have been successfully completed, but some of the fundamental properties of $\mathrm{In}_{2} \mathrm{O}_{3}$ (IO) are only just emerging. The origin of a rather high free-electron concentration in as-grown thin films, for instance, is a subject of discussion. On the one hand, interstitial and substitutional hydrogen is suggested as a shallow donor in indium oxide. ${ }^{6,7}$ On the other hand, first-principles orbital molecular calculations point to interstitial indium and oxygen vacancies as responsible for IO's high conductivity. ${ }^{8}$ Recent results of combined electronic-structure calculations and direct transport measurements show that high carrier concentration in as-grown IO films likely comes from a large density of surface donors. ${ }^{9}$ Existing models are inconclusive, but it is clear that native defects are relevant as donors and acceptors in transparent oxides.

Most of the experimental information for indium oxide is obtained for polycrystalline thin films whose properties are highly growth specific. Polycrystalline thin films are made of small crystallites joined by grain boundaries (GBs). These give rise to charged interface states and, consequently, to GB potential barriers. The existence of such barriers, with

${ }^{a)}$ Deceased. surface-like structure, is generic to many semiconducting systems and affects, to a large extent, their electronic transport. ${ }^{10}$ In particular, impurities tend to segregate at GBs, where they act as charge trapping states. It has been found, for instance, that the electrical behavior of boundaries in silicon is almost entirely dependent of the concentration of hydrogen and oxygen which is segregated to interfaces. ${ }^{11}$ Some essential properties of varistors also rely on impurities at grain boundaries. ${ }^{12}$

Knowledge of the structure and chemistry of surface defects is therefore very important for tailoring properties of semiconductor devices. Indium oxide, which crystallizes in a cubic bixbyite structure, is particularly prone to segregation of oxygen at the GB surfaces. This is brought about by the unique crystal-defect structure of bixbyite based materials. It is derived from the fluorite structure, with one fourth of the oxygen atoms removed. These so-called structural vacancies are actually empty interstitial positions which serve as fast oxygen diffusion paths and favor easy incorporation of oxygen ions. ${ }^{13}$ More interstitial oxygen ions gives rise to larger density of electronic interface states which, when charged, may lead to a "type inversion," whereby the surface region becomes hole rich, even though the grain's interior is electron rich. ${ }^{14}$

In this paper, we show that microstructure as well as the properties of surface defects crucially affect the electrical behavior of thin-film IO homo-junctions. Although usually obtained as an $n$-type material, $\mathrm{In}_{2} \mathrm{O}_{3}$ thin films deposited by sputtering under $\mathrm{O}_{2}$-rich conditions show $p$-type conduction. ${ }^{15,16}$ This enabled us to fabricate IO $p$ - $n$ homo-junctions with rectifying behavior. In order to elucidate the physical mechanism behind this, we have performed a careful structural characterization of these junctions, in addition to $\mathrm{x}$-ray 
photoelectron spectroscopy and electrical measurements. Our experimental results show the morphology of the junctions changes significantly with the growth temperature. This is important for their electrical behavior since only junctions grown at about $350{ }^{\circ} \mathrm{C}$ are rectifying. On the other hand, the segregation of oxygen ions at the GBs is most likely responsible for the formation of inversion layers in $\mathrm{O}_{2}$-rich films and their apparent $p$-type $d c$ conduction.

\section{EXPERIMENT}

We have grown indium oxide thin films by de magnetron sputtering from a ceramic $\operatorname{In}_{2} \mathrm{O}_{3}$ target in a vacuum system with a base pressure of $\sim 4 \times 10^{-6}$ Torr. Fused-quartz and heavily doped Si wafers have been used as substrates, which were kept at a constant temperature between 200 and $700{ }^{\circ} \mathrm{C}$. The total gas pressure during deposition was fixed at $1.0 \times 10^{-3}$ Torr. The deposition rate under oxygen-rich conditions was $\sim 0.1 \AA / \mathrm{s}$, at a substrate-target distance of $35 \mathrm{~cm}$. We have fabricated $\mathrm{In}_{2} \mathrm{O}_{3} p-n$ homo-junctions by sequential growth of an approximately $400 \mathrm{~nm}$ thick $n$-type layer (in a pure $\mathrm{Ar}$ ambient) and a $200 \mathrm{~nm}$ thick $p$-type $\mathrm{In}_{2} \mathrm{O}_{3}$, obtained by deposition in pure $\mathrm{O}_{2}$ atmosphere (oxygen-rich conditions). The reversed deposition order (first a $p$-type $\mathrm{In}_{2} \mathrm{O}_{3}$ layer followed by an $n$-type one) has been applied as well. Ohmic contacts to the layers were done using sputtered Au or thermally deposited In. For junctions grown on a quartz substrate, the surface layer was partially etched until the underlying one was exposed in order to contact it. Current-voltage $(I-V)$ curves were measured by a standard method. Capacitance-voltage $(C-V)$ measurements were carried out with a precision impedance analyzer in the frequency range from $40 \mathrm{~Hz}$ to $1 \mathrm{MHz}$.

The structural characterization of homo-junctions was carried out using $\mathrm{X}$-ray diffraction (XRD), scanning electron microscopy (SEM), and atomic force microscopy (AFM).
X-ray photoelectron spectroscopy (XPS) was used to identify the chemical bonding states of In and $\mathrm{O}$ atoms, to check homogeneity, and to study how the oxygen concentration varies with depth in these films. Monochromatic Al $(1486.6 \mathrm{eV})$ radiation was employed as excitation. XPS spectra, in form of wide-energy scans and high-resolution data in regions corresponding to In $3 d, \mathrm{O} 1 s$, and $\mathrm{C} 1 s$, were obtained for several thin-film junctions. To avoid charging effects, an electron flood gun has been used. The binding energies were corrected using the $\mathrm{C} 1 s$ signal at $284.6 \mathrm{eV}$ as reference. The experimental uncertainty in the energy determination was $\sim 0.10 \mathrm{eV}$. The XPS spectra have been measured in the asreceived state and after Ar-ion etching cycles which were repeated several times. The etching rate in our study was approximately $2 \mathrm{~nm} / \mathrm{min}$. Therefore, the actual boundary between two different layers in the junction could be reached after several hours of etching. We found that surface contamination, observed in initial spectra, disappears completely after ion etching for three minutes.

\section{RESULTS AND DISCUSSION}

Figure 1 shows cross-sectional SEM images and corresponding XRD patterns for junctions grown at temperatures between 250 and $700^{\circ} \mathrm{C}$. Two structures are exhibited for each deposition temperature. All diffraction peaks are indexed to a cubic bixbyite $\mathrm{In}_{2} \mathrm{O}_{3}$ phase. No peaks of impurities or other indium-related secondary phases are observed. For growth temperatures lower than $600{ }^{\circ} \mathrm{C}$, a strong (222) peak in the patterns for $\mathrm{In}_{2} \mathrm{O}_{3}$ films implies that they are preferentially textured along the (111) direction. Films obtained at higher temperatures are less textured than the ones grown at lower temperatures but have larger grain size as expected. Our studies show that the formation of junctions depends on the growth temperature, which crucially affects the films' microstructure. At the optimal temperature $\left(350^{\circ} \mathrm{C}\right)$, the films grow

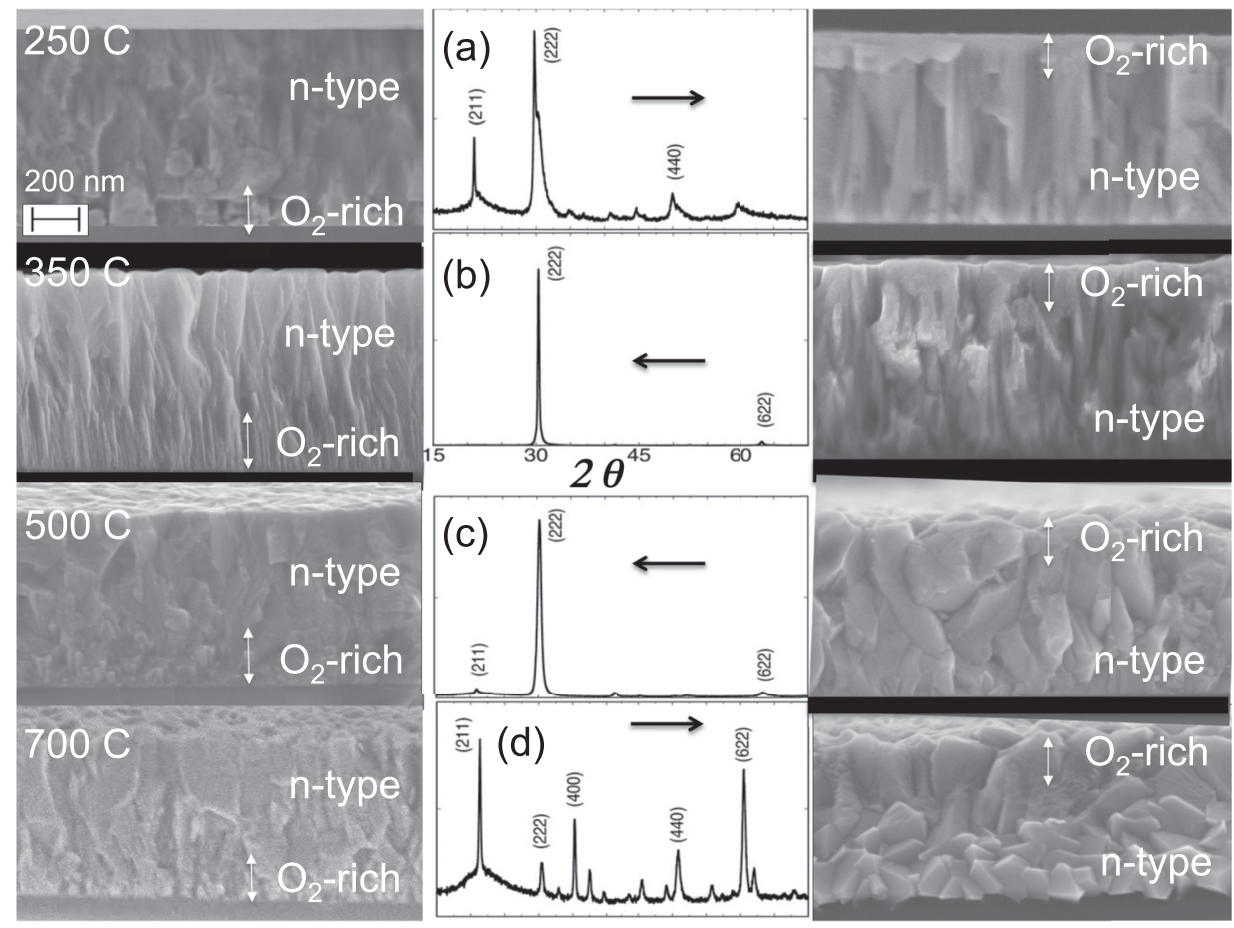

FIG. 1. Cross-sectional SEM images (on the left and on the right) and corresponding XRD patterns (center) for junctions grown at a substrate (fused quartz) temperature of (a) $250^{\circ} \mathrm{C}$; (b) $350{ }^{\circ} \mathrm{C}$; (c) $500^{\circ} \mathrm{C}$; and (d) $700^{\circ} \mathrm{C}$. The thickness of the $\mathrm{O}_{2}$-rich layer is approximately $200 \mathrm{~nm}$ in all depositions. 


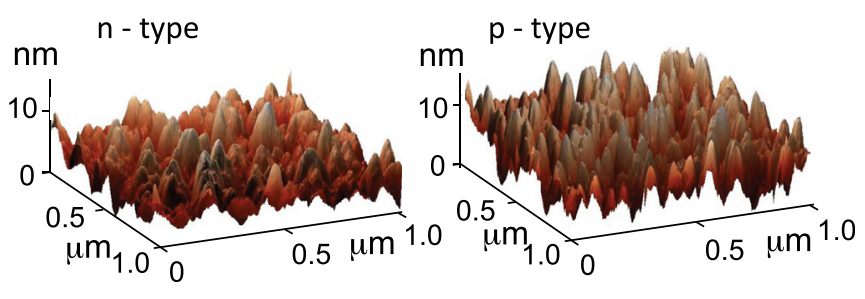

FIG. 2. Surface topography of $n$ - and $p$-type films grown at $350^{\circ} \mathrm{C}$ as probed by AFM.

in a columnar structure with a (111) preferred orientation, even in a pure Ar atmosphere (Fig. 1(b)). The cross-sectional SEM image of the junction reveals a clear boundary between $p$ - and $n$-type polycrystalline layers. However, the order of deposition also affects the junction microstructure. Layers with an $n$ - $\mathrm{In}_{2} \mathrm{O}_{3} / p$ - $\mathrm{In}_{2} \mathrm{O}_{3}$ structure show stronger preferred orientation than the $p$ - $\mathrm{In}_{2} \mathrm{O}_{3} / n$ - $\mathrm{In}_{2} \mathrm{O}_{3}$ layers. The mean grain size, obtained from SEM images, for the films grown in a $\mathrm{O}_{2}$ environment, is $19 \pm 3 \mathrm{~nm}$, while the $n$-type layer is made up of much larger grains, typically of $60 \pm 5 \mathrm{~nm}$. The junctions obtained at a lower temperature, shown in Fig. 1(a), still preserve a columnar structure and distinct pattern for two types of layers, but their growth is more disoriented. For growth temperatures higher than $350^{\circ} \mathrm{C}$, the boundary between two layers is not clear and columnar growth disappears giving rise to completely different microstructures (Figs. 1(c) and 1(d)). It is worth noting that no amorphous phase is formed at the initial stage of the growth. The interface region between the amorphous quartz substrate and the $\mathrm{In}_{2} \mathrm{O}_{3}$ film, although not exactly flat, is abrupt for all films studied. How the topography of films varies with the type of top layer is shown in Fig. 2 . The AFM image to the left is for an $n$-type layer deposited on a $p$-type but in the image to the right a $p$-type film is the top layer. Both junctions were grown at $350^{\circ} \mathrm{C}$ on a quartz substrate. The calculated $\mathrm{rms}$ and average roughness for an $5 \times 5 \mu \mathrm{m}^{2}$ area are 0.8 and $0.6 \mathrm{~nm}$, respectively, for the $n$-type film. These values are in line with those of the best IO films grown by sputtering. ${ }^{17}$ The $p$-type top layer is considerably more irregular, with 2.1 and $2.6 \mathrm{~nm}$ for the rms and average roughness, respectively.

To verify how the oxygen concentration inside the films varies with the deposition conditions, we use results from XPS measurements. Fig. 3 shows spectra obtained for a junction in which the upper $n$-type layer has been removed by etching from one half of the sample surface. Therefore, we could perform depth profiling in both layers concurrently. The core levels of In show that the binding energy of In $3 d_{5 / 2}$ stays at $444.5 \pm 0.2 \mathrm{eV}$, regardless of etching periods or type of layer. This is for indium in the formal valence state $3+.{ }^{18}$ On the other hand, the O $1 s$ spectra show broad structures. Their deconvolution yields three or four Gaussian peaks for as-deposited films, but only two peaks are resolved after films are etched for over than $3 \mathrm{~min}$. A double structure of the O $1 s$ spectra, observed in XPS studies of various metal oxides, suggests that cations of the system are in the mixed valence states. ${ }^{19}$ The main peak (OI), at about $530 \mathrm{eV}$, can be attributed to the O-In bonding state, ${ }^{19}$ in which the In atoms form $\mathrm{InO}_{6}$ octahedra with the six nearest $\mathrm{O}^{2+}$ - ions. The higher-energy OII peak is most likely related to the O-In bonding state in the oxygen-deficient region and, therefore, is more sensitive to the oxygen content. ${ }^{19}$ Alternatively, the OII peak can be attributed to oxygen in the $\mathrm{OH}$ groups or to the intermediate state between $\mathrm{O}^{2-}$ and dissociated oxygen. Such interpretation is however less plausible as variations in the intensity of this component are related to changes in the concentration of oxygen vacancies. How the total oxygen content varies with depth in one of the thin-film homo-junctions is shown in Fig. 4. It is higher for the p-type layer as expected from the growth conditions. This observation is also confirmed by comparing the integrated areas of the OI and OII peaks. The peak ratio OII/OI is clearly larger for the layers grown in $\mathrm{O}_{2}$-rich ambient as seen in Fig. 4.

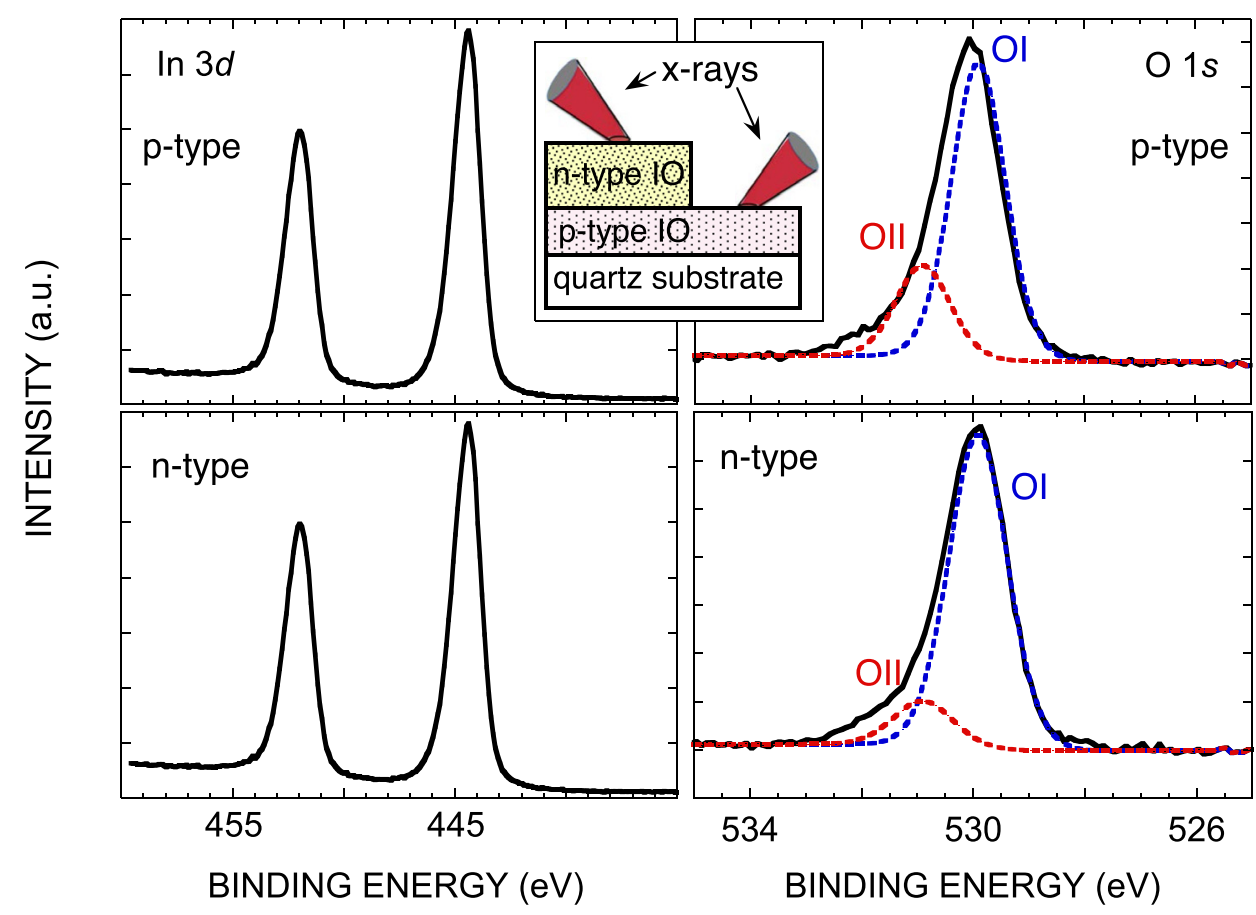

FIG. 3. XPS spectra of In $3 d$ and O $1 s$, corresponding to $p$ - and $n$-type layers, obtained for indium oxide homojunctions after $30 \mathrm{~min}$ of ion etching. The depth profile for the same junction is shown in Fig. 4(b). 


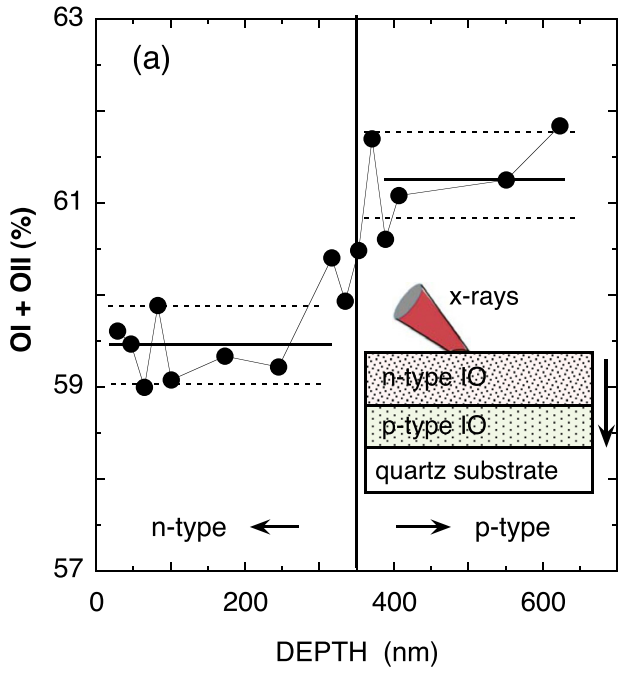

In sum, the results of micro-structural studies on thinfilm IO $p-n$ junctions show that (i) the formation of junctions depends on the growth temperature; (ii) growth temperature has a drastic effect on the films' microstructure; (iii) at optimal growth temperature of $350{ }^{\circ} \mathrm{C}$, the films show columnar structure highly textured along (111) direction; (iv) the $p$-type layers consists of much smaller grains than the $n$-type layers whose surface is also more smooth; (v) the $p$-type films show higher concentration of inter-grain oxygen, most likely coming from oxygen-rich conditions during their deposition and from much larger number of grain boundaries than in $n$-type layer.

We now turn to the results from the electrical characterization of indium oxide homo-junctions. We focus on the junctions grown at $350{ }^{\circ} \mathrm{C}$ since the ones deposited at either $250{ }^{\circ} \mathrm{C}$ or above $400^{\circ} \mathrm{C}$ show either less rectifying or nonrectifying $I-V$ curves. This behavior correlates with the structural quality of the films, in particular with abruptness of junctions which is clearly noticeable only for growth at about $350{ }^{\circ} \mathrm{C}$. Figure 5 exhibits $I-V$ curves for homojunctions grown on quartz and silicon substrates. In junctions deposited on $\mathrm{Si}$ substrate a clear rectifying response is observed, with an estimated turn-on voltage of approximately $3.0 \mathrm{eV}$ and a low (less than $5 \times 10^{-4} \mathrm{~A} / \mathrm{cm}^{2}$ at $-5 \mathrm{~V}$ ) leakage current under a reverse bias. For junctions grown on quartz substrates (curve (a) in Fig. 5), a considerably higher density of defects and dislocations leads to a larger leakage current in this case.

The forward mode of bipolar junctions is usually characterized by the ideality factor $n=(q / k T) d V / d \ln I,{ }^{20}$ where $q$ is the electron charge, $k$ is Boltzmann's constant, and $T$ is the absolute temperature. We obtain $n=19$ from the $\mathrm{In}_{2} \mathrm{O}_{3} I-V$ curves, which is comparable to the values found for other TCO $p-n$ junctions. Ideality factors greater than 2.0 , the value which is predicted by classical models of recombination of the minority carriers, ${ }^{20,21}$ can be brought about by a large number of surface states or tunneling-enhanced recombination mechanism..$^{21-23}$ The forward current tends to saturate at high values because of series resistance. Both contact and film resistances contribute to it. The series resistance is likely enhanced in our junctions by surface oxygen adsorption, which often dominate the electronic and chemical properties of metal oxide surfaces. ${ }^{24}$ This process, which usually occurs at the IO surface oxygen vacancy sites, decreases the concentration of charge carriers near the surface and gives rise to a depletion region.

To gain further insight into the physical mechanisms behind rectifying processes in the IO junctions, capacitancevoltage measurements have been performed on some devices. As discussed above, polycrystalline indium oxide consists of small grains joined by grain boundaries which give rise to interface states in the band gap. These states are charged by carriers from the interior of grains. A compensating charge in the adjacent layer (the space-charge layer) leads therefore to the formation of a potential barrier of finite width which frequently is represented as a back-to-back, or symmetric, Schottky barrier. The effect of GB barriers is negligible in $n$-type $\mathrm{In}_{2} \mathrm{O}_{3}$ films as evidenced by their ohmic behaviour.

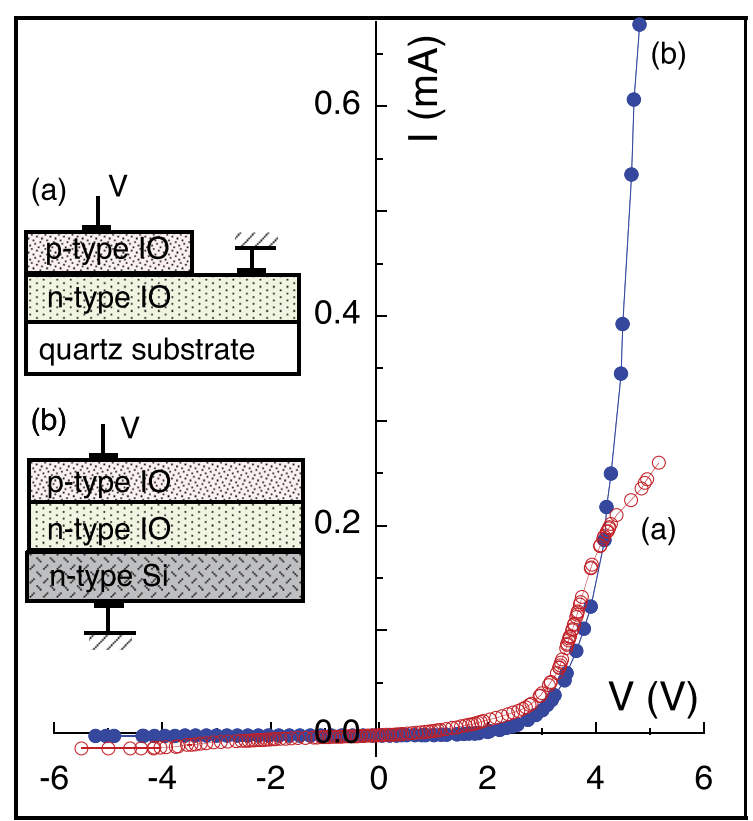

FIG. 5. $I-V$ curves for indium oxide homo-junctions deposited on (a)-quartz and (b)-Si substrate at $350^{\circ} \mathrm{C}$. 
However, the IO crystallites in films grown under $\mathrm{O}_{2}$-rich conditions are much smaller giving rise to a significantly higher density of GB interface states. Then, we infer from impedance measurements that oxygen atoms most likely segregate to GBs and capture electrons from the grains' interior. ${ }^{14}$ Consequently, $p$-type inversion layers form at the grain boundaries. A grain boundary that intersects a $p-n$ junction modifies the potential field at the junction, and at the same time have its own potential field modified by the space-charge region of the junction. This is clearly seen in $C-V$ curves shown in Fig. 6 which seem to be more typical for the $p-i-n$ device, where the total capacitance is composed of the junction and the intrinsic layer capacitance, than for the $p$ - $n$ diode with a sharp interface. ${ }^{25}$ Note that the $C-V$ curves are shifted along the voltage axis. This shift can likely be attributed to a negative fixed charge at GBs.

The total capacitance $C_{t}$ in Fig. 6 can be modeled as a series combination of the $p$-type layer capacitance $C_{i}$ and the semiconductor depletion-layer capacitance $C_{d}$ modified by the interface-trapped charge. ${ }^{25}$ An equivalent circuit, incorporating the interface-trapped charge effect, is shown in Fig. 7. In the model, $C_{s}$ and $R_{s}$ are the capacitance and resistance associated to the traps which may depend on surface potential. $R_{i}$ comes from inversion channels conduction in the layer grown under $\mathrm{O}_{2}$-rich conditions. Both $C_{i}$ and $R_{i}$ are affected by the bias voltage which reduces band bending in the conducting channels. Consequently, the resistance $R_{i}$ decreases and capacitance $C_{i}$ increases with increasing $V$ as the effective width of the space charge becomes smaller. Their variation with the applied voltage, shown in the inset of Fig. 7, is known from our previous study on $p$-type IO films. ${ }^{15}$ Therefore, we can find how the depletion capacitance varies with a reverse bias for the junctions studied. This is displayed in Fig. 7 where $\left(1 / C_{d}\right)^{2}$ is plotted versus $V$. The intercept voltage $V_{D}$, obtained form a linear fitting to the data points for $V \geq 2 \mathrm{~V}$, is approximately $1.0 \mathrm{~V}$. Therefore, $C_{d}$ varies with $V$ as expected for a bipolar junction. The depletion-layer width, calculated from the relation

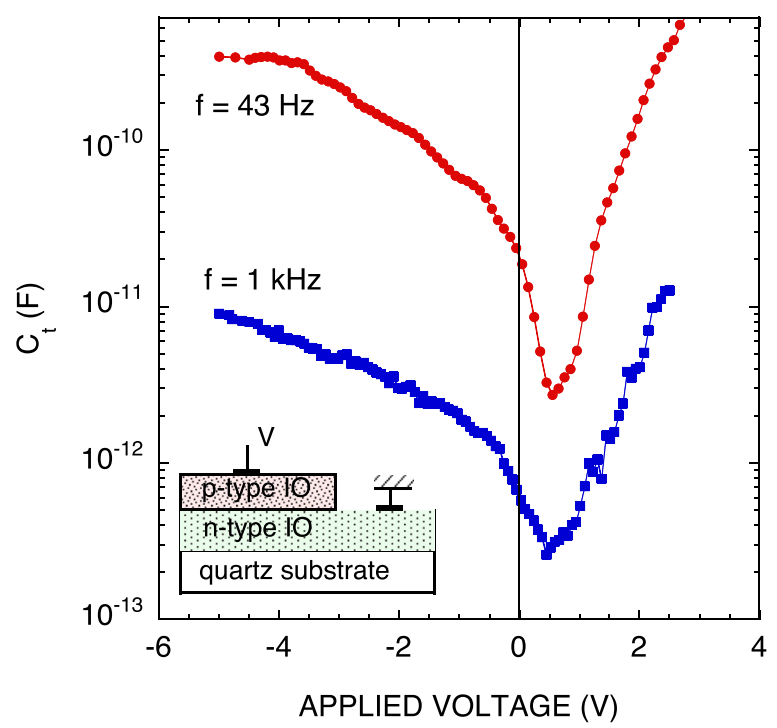

FIG. 6. $C-V$ curves at two frequencies for a IO homo-junction grown on quartz substrate at $350^{\circ} \mathrm{C}$.

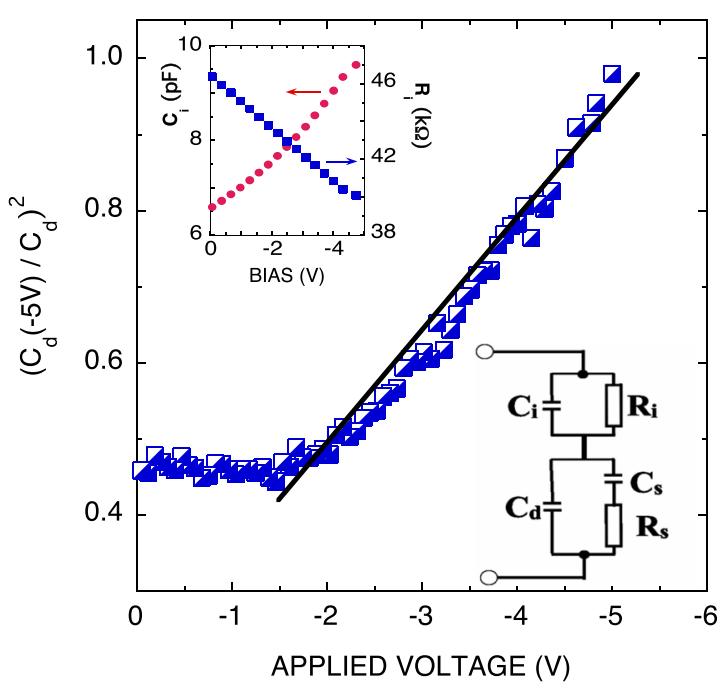

FIG. 7. Inverse of the square of the depletion capacitance versus applied voltage for a IO homo-junction grown on quartz substrate at $350^{\circ} \mathrm{C}$.

$d=\left(2 \epsilon \epsilon_{o} V_{D} / N_{A}\right)^{1 / 2}$, is approximately $40 \mathrm{~nm}$. Here, $\epsilon$ is the relative permittivity of $\operatorname{In}_{2} \mathrm{O}_{3}(\epsilon=9), \epsilon_{o}$ is the permittivity of free space, and $N_{A}$ is the acceptor concentration in the $p$ type layer, which we assume to be equal to the hole concentration obtained for $\operatorname{In}_{2} \mathrm{O}_{3}$ films grown under $\mathrm{O}_{2}$-rich conditions $\left(N_{A} \approx 2 \times 10^{17} \mathrm{~cm}^{-3}\right){ }^{15}$ The $n$-type films show much higher carrier concentration $\left(\approx 10^{20} \mathrm{~cm}^{-3}\right)$. The estimated value of $d$ agrees well with the depletion-region capacitance we measure. However, we had to assume, somehow arbitrarily, the values of $C_{s}$ and $R_{s}$ and their dependence on $V$. We used values of $2 \times 10^{8} \Omega$ and $1 \times 10^{-11} \mathrm{~F}$ for $R_{s}$ and $C_{s}$, respectively, and let the former increase and the latter decrease linearly no more than $100 \%$ with the bias up to $-5 \mathrm{~V}$.

In sum, we find that the microstructure of $\operatorname{In}_{2} \mathrm{O}_{3}$ sputtered films, which we control by the growth temperature and ambient gas, significantly affects electrical properties of junctions fabricated on these films. Rectifying homojunctions are obtained by sequential deposition of $\mathrm{O}_{2}$-rich ( $p$-type) and $n$-type layers at $350^{\circ} \mathrm{C}$. We conjecture that the segregation of oxygen ions at $\mathrm{GBs}$ in layers grown under $\mathrm{O}_{2^{-}}$ rich conditions modifies significantly the $C-V$ characteristics of the junctions. Nevertheless, the rectifying mechanism can be explained by the space-charge layer at the $p$ - $n$ interface and corresponding built-in potential. Inter-grain oxygen ions are, as well, most likely responsible for the apparent $p$-type $d c$ conduction in IO films. Such behaviour might be important in other polycrystalline thin films with a large number of interface defects at grain boundaries and might open up a range of interesting applications.

\section{ACKNOWLEDGMENTS}

We thank Carmen Cosculluela for her valuable help in growing films. We acknowledge support from grant MAT2012-38213-C02-01, from the Ministerio de Economía y Competividad of Spain. Additional support from Diputación General de Aragón (DGA-CAMRADS) and from Fondo Social Europeo (FSE) is also acknowledged. 
${ }^{1}$ I. Hamberg and C. G. Granqvist, J. Appl. Phys. 60, R123 (1986).

${ }^{2}$ B. G. Lewis and D. C. Paine, MRS Bull. 25, 22 (2000).

${ }^{3}$ C. G. Granqvist and A. Hultåker, Thin Solid Films 411, 1 (2002).

${ }^{4}$ E. Fortunato, D. Ginley, H. Hosono, and D. C. Paine, MRS Bull. 32, 242 (2007).

${ }^{5}$ T. Minami, Semicond. Sci. Technol. 20, 35 (2005).

${ }^{6}$ S. Limpijumnong, P. Reunchan, A. Janotti, and C. G. Van de Walle, Phys. Rev. B 80, 193202 (2009).

${ }^{7}$ P. D. C. King, R. L. Lichti, Y. G. Celebi, J. M. Gil, R. C. Vilo, H. V. Alberto, J. Piroto Duarte, D. J. Payne, R. G. Egdell, I. McKenzie, C. F. McConville, S. F. J. Cox, and T. D. Veal, Phys. Rev. B 80, 081201(R) (2009).

${ }^{8}$ T. Tomita, K. Yamashita, Y. Hayafuji, and H. Adachi, Appl. Phys. Lett. 87, 051911 (2005).

${ }^{9}$ S. Lany, A. Zakutayev, T. O. Mason, J. F. Wager, K. R. Poeppelmeier, J. D. Perkins, J. J. Berry, D. S. Ginley, and A. Zunger, Phys. Rev. Lett. 108, 016802 (2012).

${ }^{10}$ C. Persson and A. Zunger, Phys. Rev. Lett. 91, 266401 (2003).

${ }^{11}$ C. R. M. Grovenor, J. Phys. C 18, 4079 (1985).

${ }^{12}$ P. R. Bueno, J. A. Varela, and E. Longo, J. Eur. Ceram. Soc. 28, 505 (2008).

${ }^{13}$ G. B. Gonzlez, J. B. Cohen, J.-H. Hwang, and T. O. Mason, J. Appl. Phys. 89, 2550 (2001).
${ }^{14}$ J. Stankiewicz, M. P. Lozano, and F. Villuendas, Phys. Rev. B 85, 125306 (2012).

${ }^{15}$ J. Stankiewicz, F. Villuendas, and R. Alcalá, Appl. Phys. Lett. 96, 192108 (2010).

${ }^{16}$ S. S. Sun, P. Wu, and P. Xing, Appl. Phys. Lett. 101, 132417 (2012).

${ }^{17}$ K. C. Aw, Z. Tsakadze, A. Lohanib, and S. Mhaisalkar, Scr. Mater. 60, 48 (2009).

${ }^{18}$ C. D. Wagner and G. E. Muilenberg, Handbook of X-ray Photoelectron Spectroscopy: A Reference Book of Standard Data for Use in X-ray Photoelectron Spectroscopy (Perkin-Elmer Corp., Physical Electronics Division, 1979).

${ }^{19}$ J. C. C. Fan and J. B. Goodenough, J. Appl. Phys. 48, 3524 (1977).

${ }^{20}$ C. Sah, R. N. Noyce, and W. Shockley, Proc. IRE 45, 1228 (1957).

${ }^{21}$ J. M. Shah, Y.-L. Li, Th. Gessmann, and E. F. Schubert, J. Appl. Phys. 94, $2627(2003)$

${ }^{22}$ D. J. Dumin and G. L. Pearson, J. Appl. Phys. 36, 3418 (1965).

${ }^{23}$ U. Rau, A. Jasenek, H. W. Schock, F. Engelhard, and T. Meyer, Thin Solid Films 361-362, 298 (2000).

${ }^{24}$ V. E. Henrich, Rep. Prog. Phys. 48, 1481 (1985).

${ }^{25}$ S. M. Sze, Physics of Semiconductor Devices, 2nd ed. (Wiley, New York, 1981). 